\title{
Extreme Points and Rotundity in Musielak-Orlicz-Bochner Function Spaces Endowed with Orlicz Norm
}

\author{
Shaoqiang Shang, ${ }^{1}$ Yunan Cui, ${ }^{2}$ and Yongqiang $\mathrm{Fu}^{1}$ \\ ${ }^{1}$ Department of Mathematics, Harbin Institute of Technology, Harbin 150001, China \\ ${ }^{2}$ Department of Mathematics, Harbin University of Science and Technology, Harbin 150080, China
}

Correspondence should be addressed to Shaoqiang Shang, sqshang@163.com

Received 17 June 2010; Accepted 27 August 2010

Academic Editor: Paul Eloe

Copyright (C) 2010 Shaoqiang Shang et al. This is an open access article distributed under the Creative Commons Attribution License, which permits unrestricted use, distribution, and reproduction in any medium, provided the original work is properly cited.

The criteria for extreme point and rotundity of Musielak-Orlicz-Bochner function spaces equipped with Orlicz norm are given. Although criteria for extreme point of Musielak-Orlicz function spaces equipped with the Orlicz norm were known, we can easily deduce them from our main results.

\section{Introduction}

Let $(X,\|\cdot\|)$ be a real Banach space. $S(X)$ and $B(X)$ denote the unit sphere and unit ball, respectively. By $X^{*}$ denote the dual space of $X$. Let $N, R$, and $R^{+}$denote the set natural number, reals, and nonnegative reals, respectively.

A point $x \in A$ is said to be extreme point of $A$ if $2 x=y+z$ and $y, z \in A$ imply $y=z$. The set of all extreme points of $A$ is denoted by $\operatorname{Ext} A$. If $\operatorname{Ext} B(X)=S(X)$, then $X$ is said to be rotund. A point $x \in S(X)$ is said to be strongly extreme point if for any $\left\{x_{n}\right\}_{n=1}^{\infty},\left\{y_{n}\right\}_{n=1}^{\infty} \in X$ with $\left\|x_{n}\right\| \rightarrow 1,\left\|y_{n}\right\| \rightarrow 1$, and $x=(1 / 2)\left(x_{n}+y_{n}\right)$, there holds $\left\|x_{n}-y_{n}\right\| \rightarrow 0(n \rightarrow \infty)$. If the set of all strongly extreme points of $B(X)$ is equal to $S(X)$, then $X$ is said to be midpoint local uniform rotund.

The notion of extreme point plays an important role in some branches of mathematics. For example, the Krein-Milman theorem, Choquet integral representation theorem, Rainwater theorem on convergence in weak topology, Bessaga-Pelczynski theorem, and Elton test unconditional convergence are strongly connected with this notion. In [1], using the principle of locally reflexivity, a remarkable theorem describing connections between extreme points of $S(X)$ and strongly extreme points of $S(X)$ is proved. Namely, a Banach space $X$ is midpoint 
local uniformly rotund if and only if every point of $S(X)$ is an extreme point in $X^{* *}$. Another proof of this theorem based on Goldstein's theorem is given in [2]. Analyzing the proof of this fact one can easily see its local version, namely, if $x \in S(X)$ is a strongly extreme point in $X$, then $\kappa(x)$ is an extreme point in $X^{* *}$, where $\kappa$ is the mapping of canonical embedding of $X$ into $X^{* *}$.

The criteria for extreme point and rotundity in the classical Musielak-Orlicz function spaces have been given in [3] already. However, because of the complication of MusielakOrlicz-Bochner function spaces equipped with Orlicz norm, at present, the criteria for extreme point and rotundity have not been discussed yet. The aim of this paper is to give criteria for extreme point and rotundity of Musielak-Orlicz-Bochner function spaces equipped with Orlicz norm. By the result of this paper, it is easy to see that the result of [3] is true.

Let $\left(T, \sum, \mu\right)$ be nonatomic measurable space. Suppose that a function $M: T \times[0, \infty) \rightarrow$ $[0, \infty]$ satisfies the following conditions:

(1) for $\mu$-a.e, $t \in T, M(t, 0)=0, \lim _{u \rightarrow \infty} M(t, u)=\infty$ and $M\left(t, u^{\prime}\right)<\infty$ for some $u^{\prime}>0$;

(2) for $\mu$-a.e, $t \in T, M(t, u)$ is convex on $[0, \infty)$ with respect to $u$;

(3) for each $u \in[0, \infty), M(t, u)$ is a $\mu$-measurable function of $t$ on $T$.

Let $p(t, u)$ denote the right derivative of $M(t, \cdot)$ at $u \in R^{+}$(where if $M(t, u)=\infty$, let $p(t, u)=$ $\infty$ ) and let $q(t, \cdot)$ be the generalized inverse function of $p(t, \cdot)$ defined on $R^{+}$by

$$
q(t, v)=\sup _{u \geq 0}\{u \geq 0: p(t, u) \leq v\}
$$

Then $N(t, v)=\int_{0}^{v} q(t, s) d s$ for any $v \in R$ and $\mu$-a.e. $t \in T$. It is well known that there holds the Young inequality $u v \leq M(t, u)+N(t, v)$ for $\mu$-a.e. $t \in T$. And $u v=M(t, u)+N(t, u) \Leftrightarrow$ $u=q(t, v)$ or $v=q(t, u)$. Let

$$
e(t)=\sup \{u>0: M(t, u)=0\}, \quad E(t)=\sup \{u>0: M(t, u)<\infty\} .
$$

For fixed $t \in T$ and $v \geq 0$, if there exists $\epsilon \in(0,1)$ such that

$$
M(t, v)=\frac{1}{2} M(t, v+\varepsilon)+\frac{1}{2} M(t, v-\varepsilon)<\infty,
$$

then we call $v$ a nonstrictly convex points of $M$ with respects to $t$. The set of all nonstrictly convex point of $M$ with respect to $t$ is denoted by $K_{t}$.

For fixed $t \in T$, if $K_{t}=\Phi$, then we call that $M(t, u)$ is strictly convex with respect $u$ for $t$.

Moreover, for a given Banach space $(X,\|\cdot\|)$, we denote by $X_{T}$ the set of all strongly $\mu$-measurable functions from $T$ to $X$, and for each $u \in X_{T}$, define the modular of $u$ by

$$
\rho_{M}(u)=\int_{T} M(t,\|u(t)\|) d t
$$


Put

$$
L_{M}^{0}(X)=\left\{u \in X_{T}: \rho_{M}(\lambda u)<\infty \text { for some } \lambda>0\right\}
$$

Then the Musielak-Orlicz-Bochner function space

$$
\|u\|^{0}=\inf _{k>0} \frac{1}{k}\left[1+\rho_{M}(k u)\right]
$$

is Banach space. If $X=R, L_{M}^{0}(R)$ is said to be Musielak-Orlicz function space. Set

$$
K(u)=\left\{k>0: \frac{1}{k}\left(1+\rho_{M}(k u)\right)=\|u\|^{0}\right\} .
$$

In particular, the set $K(u)$ can be nonempty. To show that, we give a proposition.

Proposition 1.1. If $\lim _{u \rightarrow \infty}(M(t, u) / u)=\infty \mu$-a.e. $t \in T$, then $K(u) \neq \phi$ for any $u \in L_{M}^{0}(X)$.

Proof. For any $u \in L_{M}^{0}(X)$, there exists $a>0$ such that $\mu T_{0}>0$, where $T_{0}=\{t \in T:\|u(t)\| \geq a\}$. It is easy to see that $T_{0}=\cup_{n=1}^{\infty} G_{n}$, where

$$
G_{n}=\left\{t \in T_{0}: \frac{M(t, v)}{v} \geq \frac{3\|u\|^{0}}{a \cdot \mu T_{0}}, v \geq n\right\} .
$$

Noticing that $G_{1} \subset G_{2} \subset \cdots \subset G_{n} \subset \cdots$, then $\lim _{n \rightarrow \infty} \mu G_{n}=\mu T_{0}$. Hence there exists $n_{1}$ such that $\mu G_{n_{1}}>(1 / 2) \mu T_{0}$. This means that if $k>n_{1} / a$, we have

$$
\begin{array}{r}
\frac{1}{k}\left[1+\int_{T} M(t, k\|u(t)\|) d t\right] \geq \int_{G_{n_{1}}} \frac{M(t, k\|u(t)\|)}{k} d t \geq \int_{G_{n_{1}}} \frac{M(t, k a)}{k} d t \\
=a \int_{G_{n_{1}}} \frac{M(t, k a)}{k a} d t \geq a \int_{G_{n_{1}}} \frac{M\left(t, n_{1}\right)}{n_{1}} d t \geq a \frac{3\|u\|^{0}}{a \cdot \mu T_{0}} \cdot \mu G_{n_{1}}>\frac{3}{2}\|u\|^{0}
\end{array}
$$

This implies that if $\left(1 / k_{n}\right)\left(1+\rho_{M}\left(k_{n} u\right)\right) \rightarrow\|u\|^{0}(n \rightarrow \infty)$, then sequence $\left\{k_{n}\right\}_{n=1}^{\infty}$ is bounded. Without loss of generality, we may assume that $k_{n} \rightarrow k_{0}$. Without loss of generality, we may assume that $k_{1} \leq k_{2} \leq \cdots k_{n} \leq \cdots \leq k_{0}$ or $k_{1} \geq k_{2} \geq \cdots k_{n} \geq \cdots \geq k_{0}$. If $k_{1} \leq k_{2} \leq \cdots k_{n} \leq \cdots \leq$ $k_{0}$, by Levi theorem, we have

$$
\begin{aligned}
\lim _{n \rightarrow \infty} \frac{1}{k_{n}}\left[1+\rho_{M}\left(k_{n} u\right)\right] & =\lim _{n \rightarrow \infty} \frac{1}{k_{n}}+\lim _{n \rightarrow \infty} \int_{T} \frac{M\left(t,\left\|k_{n} u(t)\right\|\right)}{k_{n}} d t \\
& =\frac{1}{k_{0}}+\int_{T} \frac{M\left(t,\left\|k_{0} u(t)\right\|\right)}{k_{0}} d t \\
& =\frac{1}{k_{0}}\left[1+\rho_{M}\left(k_{0} u\right)\right] .
\end{aligned}
$$


If $k_{1} \geq k_{2} \geq \cdots k_{n} \geq \cdots \geq k_{0}$, by dominated convergence theorem, we have

$$
\begin{aligned}
\lim _{n \rightarrow \infty} \frac{1}{k_{n}}\left[1+\rho_{M}\left(k_{n} u\right)\right] & =\lim _{n \rightarrow \infty} \frac{1}{k_{n}}+\lim _{n \rightarrow \infty} \int_{T} \frac{M\left(t,\left\|k_{n} u(t)\right\|\right)}{k_{n}} d t \\
& =\frac{1}{k_{0}}+\int_{T} \frac{M\left(t,\left\|k_{0} u(t)\right\|\right)}{k_{0}} d t \\
& =\frac{1}{k_{0}}\left[1+\rho_{M}\left(k_{0} u\right)\right] .
\end{aligned}
$$

Therefore $\left(1 / k_{n}\right)\left[1+\rho_{M}\left(k_{n} u\right)\right] \rightarrow\left(1 / k_{0}\right)\left[1+\rho_{M}\left(k_{0} u\right)\right](n \rightarrow \infty)$, namely, $\left(1 / k_{0}\right)[1+$ $\left.\rho_{M}\left(k_{0} u\right)\right]=\|u\|^{0}$. This implies $k_{0} \in K(u)$.

\section{Main Results}

In order to obtain the main theorems of this paper, we first give some lemmas.

Lemma 2.1. If $K(u)=\phi$, then $\|u\|^{0}=\int_{T} A(t) \cdot\|u(t)\| d t$, where $A(t)=\lim _{u \rightarrow \infty}(M(t, u) / u)$.

Proof. By proof of Proposition 1.1, we know that if $K(u)=\phi$, then there exists $\left\{k_{n}\right\}_{n=1}^{\infty}$ such that $\left(1 / k_{n}\right)\left(1+\rho_{M}\left(k_{n} u\right)\right) \rightarrow\|u\|^{0}$ and $k_{n} \rightarrow \infty$ as $n \rightarrow \infty$. Without loss of generality, we may assume that $k_{1} \leq k_{2} \leq \cdots k_{n} \leq \cdots$. By Levi theorem, we have

$$
\begin{aligned}
\|u\|^{0} & =\lim _{n \rightarrow \infty} \frac{1}{k_{n}}\left(1+\int_{T} M\left(t, k_{n}\|u(t)\|\right) d t\right) \\
& =\lim _{n \rightarrow \infty} \frac{1}{k_{n}}\left(1+\int_{\{t \in T:\|u(t)\| \neq 0\}} M\left(t, k_{n}\|u(t)\|\right) d t\right) \\
& =\lim _{n \rightarrow \infty} \frac{1}{k_{n}}+\lim _{n \rightarrow \infty} \int_{\{t \in T:\|u(t)\| \neq 0\}} \frac{M\left(t, k_{n}\|u(t)\|\right)}{k_{n}\|u(t)\|} \cdot\|u(t)\| d t \\
& =\int_{\{t \in T:\|u(t)\| \neq 0\}} \lim _{n \rightarrow \infty} \frac{M\left(t, k_{n}\|u(t)\|\right)}{k_{n}\|u(t)\|} \cdot\|u(t)\| d t \\
& =\int_{\{t \in T:\|u(t)\| \neq 0\}} A(t) \cdot\|u(t)\| d t \\
& =\int_{T} A(t) \cdot\|u(t)\| d t .
\end{aligned}
$$

Hence the conclusion of the lemma is true. 
Lemma 2.2. If the set $K(u)$ consists of one element from $(0,+\infty)$, then $\|u\|^{0}<\int_{T} A(t) \cdot\|u(t)\| d t$.

Proof. Pick $k_{1}>k_{2}>0$; then we have

$$
\begin{aligned}
& \rho_{M}\left(k_{1} u \chi_{E_{n}}\right) \geq \int_{T} k_{1}\left\|u(t) \chi_{E_{n}}(t)\right\| \cdot p\left(t,\left\|k_{2} u(t) \chi_{E_{n}}(t)\right\|\right) d t-\rho_{N}\left(p\left(k_{2} u \chi_{E_{n}}\right)\right), \\
& \rho_{M}\left(k_{2} u \chi_{E_{n}}\right)=\int_{T} k_{2}\left\|u(t)_{X_{n}}(t)\right\| \cdot p\left(t,\left\|k_{2} u(t)_{X_{n}}(t)\right\|\right) d t-\rho_{N}\left(p\left(k_{2} u \chi_{E_{n}}\right)\right),
\end{aligned}
$$

where

$$
E_{n}=\left\{t \in T: k_{2}\|u(t)\| \leq n, p\left(t,\left\|k_{2} u(t)\right\|\right) \leq n\right\} .
$$

It follows that

$$
\begin{aligned}
\frac{1}{k_{1}}[1 & \left.+\rho_{M}\left(k_{1} u \chi_{E_{n}}\right)\right]-\frac{1}{k_{2}}\left[1+\rho_{M}\left(k_{2} u_{\chi_{E_{n}}}\right)\right] \\
= & \frac{k_{1}-k_{2}}{k_{1} k_{2}}\left(-1+\frac{k_{2}}{k_{1}-k_{2}}\left[\rho_{M}\left(k_{1} u \chi_{E_{n}}\right)-\rho_{M}\left(k_{2} u \chi_{E_{n}}\right)\right]-\rho_{M}\left(k_{2} u \chi_{E_{n}}\right)\right) \\
\geq & \frac{k_{1}-k_{2}}{k_{1} k_{2}}\left(-1+\frac{k_{2}}{k_{1}-k_{2}} \int_{T}\left(k_{1}-k_{2}\right)\left\|u(t) \chi_{E_{n}}(t)\right\| \cdot p\left(t,\left\|k_{2} u(t) \chi_{E_{n}}(t)\right\|\right) d t\right. \\
& \left.-\rho_{M}\left(k_{2} u \chi_{E_{n}}\right)\right) \\
= & \frac{k_{1}-k_{2}}{k_{1} k_{2}}\left(\rho_{N}\left(p\left(k_{2} u \chi_{E_{n}}\right)\right)-1\right) .
\end{aligned}
$$

Let $n \rightarrow \infty$; then we obtain

$$
\frac{1}{k_{1}}\left[1+\rho_{M}\left(k_{1} u \chi_{E}\right)\right] \geq \frac{k_{1}-k_{2}}{k_{1} k_{2}}\left(\rho_{N}\left(p\left(k_{2} u \chi_{E}\right)\right)-1\right)+\frac{1}{k_{2}}\left[1+\rho_{M}\left(k_{2} u_{\chi E}\right)\right],
$$

where

$$
E=\left\{t \in T: p\left(t,\left\|k_{2} u(t)\right\|\right)<\infty\right\}
$$


If $p\left(t,\left\|k_{2} u(t)\right\|\right)=\infty$, then $M\left(t, k_{1}\|u(t)\|\right) \geq M\left(t, k_{2}\|u(t)\|\right)=\infty$. Hence we have

$$
\frac{1}{k_{1}}\left[1+\rho_{M}\left(k_{1} u\right)\right] \geq \frac{k_{1}-k_{2}}{k_{1} k_{2}}\left(\rho_{N}\left(p\left(k_{2} u\right)\right)-1\right)+\frac{1}{k_{2}}\left[1+\rho_{M}\left(k_{2} u\right)\right]
$$

Moreover, there exists $k_{0} \in R^{+}$such that $\rho_{N}(p(k u)) \geq 1$, whenever $k \geq k_{0}$. This means that function $F(k)=(1 / k)\left[1+\rho_{M}(k u)\right]$ is nondecreasing, when $n$ is large enough. Pick sequence $\left\{k_{n}\right\}_{n=1}^{\infty}$ such that $0<k_{1}<k_{2} \cdots<k_{n}<\cdots$. By Levi theorem, we have

$$
\begin{aligned}
\int_{T} A(t) \cdot\|u(t)\| d t & =\int_{T} \lim _{n \rightarrow \infty} \frac{M\left(t, k_{n}\|u(t)\|\right)}{k_{n}\|u(t)\|} \cdot\|u(t)\| d t \\
& =\lim _{n \rightarrow \infty} \int_{\mathrm{T}} \frac{M\left(t, k_{n}\|u(t)\|\right)}{k_{n}\|u(t)\|} \cdot\|u(t)\| d t \\
& =\lim _{n \rightarrow \infty} \frac{1}{k_{n}} \int_{T} M\left(t, k_{n}\|u(t)\|\right) d t \\
& =\lim _{n \rightarrow \infty} \frac{1}{k_{n}}\left[1+\int_{T} M\left(t, k_{n}\|u(t)\|\right) d t\right] \\
& >\frac{1}{l}\left[1+\int_{T} M(t, l\|u(t)\|) d t\right]=\|u\|^{0},
\end{aligned}
$$

where $\{l\}=K(u)$. Hence the conclusion of the lemma is true.

Lemma 2.3 (see [3]). Let $L_{M}^{0}(R)$ be rotund, then $M(t, u)$ is strictly convex with respect to $u$ for almost all $t \in T$.

Theorem 2.4. Let $L_{M}^{0}(X)$ be Musielak-Orlicz-Bochner function spaces, then $u \in S\left(L_{M}^{0}(X)\right)$ is an extreme point of $B\left(L_{M}^{0}(X)\right)$ if and only if

(a) the set $K(u)$ consists of one element from $(0,+\infty)$;

(b) $v, w \in X_{T}$ with $u=\lambda v+(1-\lambda) w$ and $\|u(t)\|=\|v(t)\|=\|w(t)\| \mu$-a.e that on $T$ implies $v=w$, where $\lambda \in(0,1)$;

(c) $\mu\left\{t \in T: k\|u(t)\| \in K_{t}\right\}=0$, where $k \in K(u)$.

Proof. Necessity. (a1) Suppose that $u$ is an extreme point of the unit ball $B\left(L_{M}^{0}\right)$ and $K(u)=\phi$, then $\|u\|^{0}=\int_{T} A(t) \cdot\|u(t)\| d t$ by Lemma 2.1. Decompose $T$ into $T_{1}$ and $T_{2}$ such that $\int_{T_{1}} A(t)$. $\|u(t)\| d t=\int_{T_{2}} A(t) \cdot\|u(t)\| d t$. Pick $\varepsilon \in(0,1)$. Put

$$
\begin{aligned}
& u_{1}(t)=(u(t)+\varepsilon u(t)) \chi_{T_{1}}+(u(t)-\varepsilon u(t)) \chi_{T_{2}}, \\
& u_{2}(t)=(u(t)-\varepsilon u(t)) \chi_{T_{1}}+(u(t)+\varepsilon u(t)) \chi_{T_{2}} .
\end{aligned}
$$


Obviously, $u=(1 / 2)\left(u_{1}+u_{2}\right)$ and $u_{1} \neq u_{2}$. Moreover, we have

$$
\begin{aligned}
\left\|u_{1}\right\|^{0} \leq & \int_{T} A(t) \cdot\left\|u_{1}(t)\right\| d t \\
= & \int_{T_{1}} A(t) \cdot\|u(t)+\varepsilon u(t)\| d t+\int_{T_{2}} A(t) \cdot\|u(t)-\varepsilon u(t)\| d t \\
= & \int_{T_{1}} A(t) \cdot\|u(t)\| d t+\int_{T_{1}} \varepsilon A(t) \cdot\|u(t)\| d t+\int_{T_{2}} A(t) \cdot\|u(t)\| d t \\
& -\int_{T_{2}} \varepsilon A(t) \cdot\|u(t)\| d t \\
= & \int_{T_{1}} A(t) \cdot\|u(t)\| d t+\int_{T_{2}} A(t) \cdot\|u(t)\| d t \\
= & \int_{T} A(t) \cdot\|u(t)\| d t \\
= & 1 .
\end{aligned}
$$

Similarly, we have $\left\|u_{2}\right\|^{0} \leq 1$. Hence $u_{1}, u_{1} \in B\left(L_{M}^{0}(X)\right)$. Therefore $u \in S\left(L_{M}^{0}(X)\right)$ is not an extreme point of $B\left(L_{M}^{0}(X)\right)$, a contradiction. Hence $K(u) \neq \phi$. Suppose that $\|u\|^{0}=\int_{T} A(t)$. $\|u(t)\| d t$. Similarly, we get a contradiction.

The necessity of (b) is obvious.

(c) Set

$$
\begin{aligned}
& H_{1}=\{t \in T: u(t)=0, e(t)>0\}, \\
& H_{2}=\{t \in T: 2 M(t, k\|u(t)\|)=M(t, k\|u(t)\|+\varepsilon)+M(t, k\|u(t)\|-\varepsilon), \quad\|u(t)\| \neq 0\},
\end{aligned}
$$

where $\{k\} \in K(u)$. Suppose that (c) does not hold. Then $\mu H_{1}>0$ or $\mu H_{2}>0$.

If $\mu H_{1}>0$, then for any $x \in S(X)$, by setting

$$
(v(t), w(t))= \begin{cases}\left(\frac{1}{2 k} e(t) x,-\frac{1}{2 k} e(t) x\right), & t \in H_{1}, \\ (u(t), u(t)), & t \in T \backslash H_{1}\end{cases}
$$

we have $u \neq w$ and $u=(1 / 2)(v+w)$. Moreover, we have

$$
\begin{aligned}
\|v\|^{0} & \leq \frac{1}{k}\left(1+\rho_{M}(k u)\right) \\
& =\frac{1}{k}\left(1+\int_{H_{1}} M\left(t, k\left\|\frac{1}{2 k} e(t)\right\|\right) d t+\int_{T \backslash H_{1}} M(t, k\|u(t)\|) d t\right) \\
& =\frac{1}{k}\left(1+\int_{T \backslash H_{1}} M(t, k\|u(t)\|) d t\right) \\
& \leq\|u\|^{0} .
\end{aligned}
$$


Similarly, we have $\|w\|^{0} \leq 1$. Hence $v, w \in B\left(L_{M}^{0}(X)\right)$. Therefore $u \in S\left(L_{M}^{0}(X)\right)$ is not an extreme point of $B\left(L_{M}^{0}(X)\right)$, a contradiction.

If $\mu H_{2}>0$, it is easy to see that $H_{2} \subset \cup_{n=1}^{\infty}\{t \in T:\|u(t)\| \neq 0, M(t, k\|u(t)\|)=$ $(1 / 2) M(t,(1+1 / n) k\|u(t)\|)+(1 / 2) M(t,(1-1 / n) k\|u(t)\|)\}$, where $\{k\} \in K(u)$. Then there exists $n_{0} \in N$ such that

$$
\begin{aligned}
& H=\{t \in T: u(t) \neq 0, \\
& \left.\quad M(t, k\|u(t)\|)=\frac{1}{2} M\left(t,\left(1+\frac{1}{n_{0}}\right) k\|u(t)\|\right)+\frac{1}{2} M\left(t,\left(1-\frac{1}{n_{0}}\right) k\|u(t)\|\right)<\infty\right\}
\end{aligned}
$$

is not a noll set. Decompose $H$ into $E$ and $F$ such that $\int_{E} p\left(t,\left(1 / n_{0}\right) k\|u(t)\|\right) d t=$ $\int_{F} p\left(t,\left(1 / n_{0}\right) k\|u(t)\|\right) d t$. Define

$$
(v(t), w(t))= \begin{cases}\left(\left(1+\frac{1}{n_{0}}\right) u(t),\left(1-\frac{1}{n_{0}}\right) u(t)\right), & t \in E, \\ \left(\left(1-\frac{1}{n_{0}}\right) u(t),\left(1+\frac{1}{n_{0}}\right) u(t)\right), & t \in F, \\ (u(t), u(t)), & t \in T \backslash(E \cup F) .\end{cases}
$$

Then $u \neq w$ and $u=(1 / 2)(v+w)$. Furthermore, we have

$$
\begin{aligned}
\|v\|^{0} \leq & \frac{1}{k}\left(1+\rho_{M}(k v)\right) \\
= & \frac{1}{k}\left(1+\int_{T \backslash(E \cup F)} M(t, k\|u(t)\|) d t+\int_{E} M\left(t,\left(1+\frac{1}{n_{0}}\right) k\|u(t)\|\right) d t\right. \\
& \left.\quad+\int_{F} M\left(t,\left(1-\frac{1}{n_{0}}\right) k\|u(t)\|\right) d t\right) \\
= & \frac{1}{k}\left(1+\int_{T \backslash(E \cup F)} M(t, k\|u(t)\|) d t+\int_{E} M(t, k\|u(t)\|) d t\right. \\
& \left.\quad+\int_{E} p\left(t, \frac{1}{n_{0}} k\|u(t)\|\right) d t+\int_{F} M(t, k\|u(t)\|) d t-\int_{F} p\left(t, \frac{1}{n_{0}} k\|u(t)\|\right) d t\right) \\
= & \frac{1}{k}\left(1+\int_{T \backslash(E \cup F)} M(t, k\|u(t)\|) d t+\int_{E} M(t, k\|u(t)\|) d t+\int_{F} M(t, k\|u(t)\|) d t\right) \\
= & \frac{1}{k}\left(1+\rho_{M}(k u)\right) \\
= & \|u\|^{0}=1 .
\end{aligned}
$$


Similarly, we have $\|w\|^{0} \leq 1$. Hence $v, w \in B\left(L_{M}^{0}(X)\right)$. Therefore $u \in S\left(L_{M}^{0}(X)\right)$ is not an extreme point, a contradiction. Hence (c) is true.

(a2) If $K(u) \neq \phi$ and $u \in S\left(L_{M}^{0}(X)\right)$ is an extreme point, suppose that there exists $k_{1}, k_{2} \in K(u)$ satisfying $k_{1} \neq k_{2}$. Define $k=k_{1} k_{2} /\left(k_{1}+k_{2}\right)$,

$$
\begin{aligned}
2 & =\|u\|^{0}+\|u\|^{0} \\
& =\frac{k_{1}+k_{2}}{k_{1} k_{2}}\left[1+\frac{k_{2}}{k_{1}+k_{2}} \rho_{M}\left(k_{1} u\right)+\frac{k_{1}}{k_{1}+k_{2}} \rho_{M}\left(k_{2} u\right)\right] \\
& =\frac{k_{1}+k_{2}}{k_{1} k_{2}}\left[1+\frac{k_{2}}{k_{1}+k_{2}} \int_{T} M\left(t,\left\|k_{1} u(t)\right\|\right) d t+\frac{k_{1}}{k_{1}+k_{2}} \int_{T} M\left(t,\left\|k_{2} u(t)\right\|\right) d t\right] \\
& \geq \frac{k_{1}+k_{2}}{k_{1} k_{2}}\left[1+\int_{T} M\left(t, \frac{k_{2}}{k_{1}+k_{2}}\left\|k_{1} u(t)\right\|+\frac{k_{1}}{k_{1}+k_{2}}\left\|k_{2} u(t)\right\|\right) d t\right] \\
& =\frac{k_{1}+k_{2}}{k_{1} k_{2}}\left[1+\int_{T} M\left(t,\left\|\frac{2 k_{1} k_{2}}{k_{1}+k_{2}} u(t)\right\|\right) d t\right] \\
& =2 \frac{1}{2 k}\left[1+\rho_{M}(2 k u)\right] \\
& \geq 2\|u\|^{0} \\
& =2 .
\end{aligned}
$$

This implies that

$$
\|u\|^{0}=\frac{1}{2 k}\left[1+\rho_{M}(2 k u)\right]
$$

(i.e., $2 k \in K(u)$ ),

$$
\frac{k_{2}}{k_{1}+k_{2}} M\left(t, k_{1}\|u(t)\|\right)+\frac{k_{1}}{k_{1}+k_{2}} M\left(t, k_{2}\|u(t)\|\right)=M(t, 2 k\|u(t)\|) .
$$

Since $k_{1}\|u(t)\| \neq k_{2}\|u(t)\|$ on $\{t \in T:\|u(t)\| \neq 0\}$, then $2 k\|u(t)\| \in K_{t}$ on $\{t \in T:\|u(t)\| \neq 0\}$, a contradiction. Therefore (a) is true. 
Sufficiency. We first prove that for $u, u_{1}, u_{2} \in S\left(L_{M}^{0}(X)\right)$ with $u=(1 / 2)\left(u_{1}+u_{2}\right)$ at least one of the sets $K\left(u_{1}\right)$ or $K\left(u_{2}\right)$ is nonempty. Suppose that $K\left(u_{1}\right)=\phi$ and $K\left(u_{2}\right)=\phi$. Hence we have

$$
\begin{aligned}
1 & =\left\|\frac{1}{2}\left(u_{1}+u_{2}\right)\right\|^{0} \\
& <\int_{T} A(t) \cdot\left\|\frac{1}{2}\left(u_{1}(t)+u_{2}(t)\right)\right\| d t \\
& \leq \frac{1}{2} \int_{T} A(t) \cdot\left\|u_{1}(t)\right\| d t+\frac{1}{2} \int_{T} A(t) \cdot\left\|u_{2}(t)\right\| d t \\
& =\frac{1}{2}\left\|u_{1}\right\|^{0}+\frac{1}{2}\left\|u_{2}\right\|^{0} \\
& =1,
\end{aligned}
$$

a contradiction. This contradiction shows that $K\left(u_{1}\right) \neq \phi$ or $K\left(u_{2}\right) \neq \phi$.

Now we will prove that $K\left(u_{1}\right) \neq \phi$ and $K\left(u_{2}\right) \neq \phi$. Otherwise, we can assume without loss of generality that $K\left(u_{1}\right) \neq \phi$ and $K\left(u_{2}\right)=\phi$. Put

$$
\left[u_{1}, u\right)=\left\{(1-\lambda) u_{1}+\lambda u: 0<\lambda<1\right\}, \quad\left(u, u_{2}\right]=\left\{(1-\lambda) u+\lambda u_{2}: 0<\lambda<1\right\} .
$$

Next we will prove that $K(y) \neq \phi$ for all $y \in\left[u_{1}, u\right)$ and $K(y)=\phi$ for all $y \in\left(u, u_{2}\right]$. Assume first for the contrary that this is $u_{3} \in\left[u_{1}, u\right)$ such that $K\left(u_{3}\right)=\phi$. Then there exists $\lambda_{3} \in[0,1)$ such that $u_{3}=\left(1-\lambda_{3}\right) u_{1}+\lambda_{3} u$. Since $u_{1}=2 u-u_{2}$, we have

$$
u_{3}=\left(1-\lambda_{3}\right)\left(2 u-u_{2}\right)+\lambda_{3} u=\left(2-\lambda_{3}\right) u-\left(1-\lambda_{3}\right) u_{2} .
$$

Hence $u=\left(1 /\left(2-\lambda_{3}\right)\right) u_{3}+\left(\left(1-\lambda_{3}\right) /\left(2-\lambda_{3}\right)\right) u_{2}$. Therefore

$$
\begin{aligned}
1=\|u\|^{0} & <\int_{T} A(t) \cdot\left\|\frac{1}{2-\lambda_{3}} u_{3}(t)+\frac{1-\lambda_{3}}{2-\lambda_{3}} u_{2}(t)\right\| d t \\
& \leq \frac{1}{2-\lambda_{3}} \int_{T} A(t) \cdot\left\|u_{3}(t)\right\| d t+\frac{1-\lambda_{3}}{2-\lambda_{3}} \int_{T} A(t) \cdot\left\|u_{2}(t)\right\| d t \\
& =\frac{1}{2-\lambda_{3}}\left\|u_{3}\right\|^{0}+\frac{1-\lambda_{3}}{2-\lambda_{3}}\left\|u_{2}\right\|^{0} \\
& =1
\end{aligned}
$$

a contradiction. 
Assume now for the contrary that this is $u_{4} \in\left(u, u_{2}\right]$ such that $K\left(u_{4}\right) \neq \phi$. We can find $u_{5} \in\left(u, u_{2}\right]$ such that $u=(1 / 2)\left(u_{4}+u_{5}\right)$ and $u_{4} \neq u_{5}$. Therefore there are $k_{4} \geq 1$ and $k_{5} \geq 1$ such that

$$
\left\|u_{4}\right\|^{0}=\frac{1}{k_{4}}\left[1+\rho_{M}\left(k_{4} u_{4}\right)\right], \quad\left\|u_{5}\right\|^{0}=\frac{1}{k_{5}}\left[1+\rho_{M}\left(k_{5} u_{5}\right)\right]
$$

By the convexity of the modular $\rho_{M}$ we have

$$
\begin{aligned}
\rho_{M}\left(\frac{2 k_{4} k_{5}}{k_{4}+k_{5}} u\right) & =\rho_{M}\left(\frac{k_{4} k_{5}}{k_{4}+k_{5}}\left(u_{4}+u_{5}\right)\right) \\
& =\rho_{M}\left(\frac{k_{5}}{k_{4}+k_{5}} k_{4} u_{4}+\frac{k_{4}}{k_{4}+k_{5}} k_{5} u_{5}\right) \\
& =\int_{T} M\left(t,\left\|\frac{k_{5}}{k_{4}+k_{5}} k_{4} u_{4}(t)+\frac{k_{4}}{k_{4}+k_{5}} k_{5} u_{5}(t)\right\|\right) d t \\
& \leq \int_{T} M\left(t, \frac{k_{5}}{k_{4}+k_{5}}\left\|k_{4} u_{4}(t)\right\|+\frac{k_{4}}{k_{4}+k_{5}}\left\|k_{5} u_{5}(t)\right\|\right) d t \\
& \leq \frac{k_{5}}{k_{4}+k_{5}} \int_{T} M\left(t,\left\|k_{4} u_{4}(t)\right\|\right) d t+\frac{k_{4}}{k_{4}+k_{5}} \int_{T} M\left(t,\left\|k_{5} u_{5}(t)\right\|\right) d t \\
& =\frac{k_{5}}{k_{4}+k_{5}} \rho_{M}\left(k_{4} u_{4}\right)+\frac{k_{4}}{k_{4}+k_{5}} \rho_{M}\left(k_{5} u_{5}\right) .
\end{aligned}
$$

Hence

$$
\begin{aligned}
2 & =2\|u\|^{0} \\
& \leq \frac{k_{4}+k_{5}}{k_{4} k_{5}}\left(1+\rho_{M}\left(\frac{k_{4} k_{5}}{k_{4}+k_{5}} 2 u\right)\right) \\
& \leq \frac{k_{4}+k_{5}}{k_{4} k_{5}}\left(1+\frac{k_{5}}{k_{4}+k_{5}} \rho_{M}\left(k_{4} u_{4}\right)+\frac{k_{4}}{k_{4}+k_{5}} \rho_{M}\left(k_{5} u_{5}\right)\right) \\
& \leq \frac{1}{k_{4}}\left(1+\rho_{M}\left(k_{4} u_{4}\right)\right)+\frac{1}{k_{5}}\left(1+\rho_{M}\left(k_{5} u_{5}\right)\right) \\
& =2 .
\end{aligned}
$$

Consequently, all inequalities from the last three lines are equalities in fact. Therefore $2\left(k_{4} \mathrm{k}_{5} /\left(k_{4}+k_{5}\right)\right)=k$ and

$$
M(t,\|k u(t)\|)=\frac{k_{5}}{k_{4}+k_{5}} M\left(t,\left\|k_{4} u_{4}(t)\right\|\right)+\frac{k_{4}}{k_{4}+k_{5}} M\left(t,\left\|k_{5} u_{5}(t)\right\|\right)
$$

for $\mu$-a.e $t \in T$. By $\mu\left\{t \in T: k\|u(t)\| \in K_{t}\right\}=0$, it follows that $\|k u(t)\|=\left\|k_{4} u_{4}(t)\right\|=\left\|k_{5} u_{5}(t)\right\|$ for $\mu$-a.e $t \in T$. And we have $k u(t)=\left(k_{5} /\left(k_{4}+k_{5}\right)\right) k_{4} u_{4}(t)+\left(k_{4} /\left(k_{4}+k_{5}\right)\right) k_{5} u_{5}(t)$ for $\mu$-a.e $t \in T$. By (b), we have $k u=k_{4} u_{4}=k_{5} u_{5}$. Since $u_{4}, u_{5}, u \in S\left(L_{M}^{0}(X)\right)$, we get $k_{4}=k_{5}=k$, which gives 
$u_{4}=u_{5}=u$. This contradicts the inequality $u_{4} \neq u_{5}$. Thus $K(y)=\phi$ for any $y \in\left(u, u_{2}\right]$. Take $u_{n}=(1-1 / n) u+(1 / n) u_{2}$ for all $n \in N$. Then $u_{n} \in\left(u, u_{2}\right]$ for all $n \in N$. Hence $K\left(u_{n}\right)=\phi$, and consequently $\left\|u_{n}\right\|^{0}=\int_{T} A(t) \cdot\left\|u_{n}(t)\right\| d t$ for all $n \in N$. Note that $\left\|u_{n}-u\right\|^{0} \rightarrow 0(n \rightarrow \infty)$ and $\lim _{n \rightarrow \infty}\left\|u_{n}(t)\right\|=\|u(t)\|$ for $\mu$-a.e, $t \in T$. Since $K(u)=\{k\}$, with $0<k<\infty$, we have

$$
\lim _{n \rightarrow \infty}\left\|u_{n}\right\|^{0}=\|u\|^{0}=\lim _{n \rightarrow \infty} \int_{T} A(t) \cdot\left\|u_{n}(t)\right\| d t \geq \int_{T} A(t) \cdot\|u(t)\| d t>\|u\|^{0},
$$

a contradiction. Therefore $K\left(u_{1}\right) \neq \phi$ and $K\left(u_{2}\right) \neq \phi$. Now repeating the same procedure as above, putting $u_{1}$ and $u_{2}$ instead of $u_{4}$ and $u_{5}$, respectively, we get

$$
k_{1} u_{1}(t)=k_{2} u_{2}(t)=k u(t)
$$

for $\mu$-a.e, $t \in T$. Hence, by the fact that $u_{1}, u_{2}, u \in S\left(L_{M}^{0}(X)\right)$, we have $k_{1}=k_{2}=k$, and consequently, $u_{1}=u_{2}=u$. Thus $u$ is an extreme point of $B\left(L_{M}^{0}(X)\right)$.

Corollary 2.5 (see [3]). $u \in S\left(L_{M}^{0}(R)\right)$ is an extreme point of $B\left(L_{M}^{0}(R)\right)$ if and only if

(a) the set $K(u)$ consists of one element from $(0,+\infty)$;

(b) $\mu\left\{t \in T: k|u(t)| \in K_{t}\right\}=0$, where $k \in K(u)$.

Finally, we investigate the rotundity of $L_{M}^{0}(X)$.

Theorem 2.6. $L_{M}^{0}(X)$ is rotund if and only if:

(a) for any $u \in S\left(L_{M}^{0}(X)\right)$, the set $K(u)$ consists of one element from $(0,+\infty)$;

(b) $X$ is rotund;

(c) $M(t, u)$ is strictly convex with respect to $u$ for almost all $t \in T$.

Proof. Sufficiency is obvious by Theorem 2.4.

Necessity. (a) is obvious by (a) of Theorem 2.4. $L_{M}^{0}(R)$ is isometrically isomorphic to closed subspace of $L_{M}^{0}(X)$, thus $L_{M}^{0}(R)$ is rotund. By Lemma 2.3, (c) is obvious.

If (b) is not true, then there exist $x, y, z \in S(X)$ with $2 x=y+z$ and $y \neq z$. Pick $h(t) \in$ $S\left(L_{M}^{0}(X)\right)$, then there exists $d>0$ such that $\mu H>0$, where $H=\{t \in T:\|h(t)\| \geq d\}$. Since $h(t) \in S\left(L_{M}^{0}(X)\right)$, then there exists $k^{\prime}>0$ such that

$$
\int_{H} M\left(t, k^{\prime}\|h(t)\|\right) d t \leq \int_{T} M\left(t, k^{\prime}\|h(t)\|\right) d t<\infty
$$

Set

$$
u(t)=d \cdot x \cdot \chi_{H}(t), \quad v(t)=d \cdot y \cdot \chi_{H}(t), \quad w(t)=d \cdot z \cdot \chi_{H}(t) .
$$

We have

$$
\int_{T} M\left(t, k^{\prime}\|u(t)\|\right) d t=\int_{H} M\left(t, k^{\prime} d\right) d t \leq \int_{H} M(t, k\|h(t)\|) d t<\infty .
$$


This implies that $u(t) \in L_{M}^{0}(X)$. Similarly, we have $v(t), w(t) \in L_{M}^{0}(X)$. It is easy to see that $\|u\|^{0}=\|v\|^{0}=\|w\|^{0}$. Then

$$
\left\|\frac{u}{\|u\|^{0}}\right\|^{0}=\left\|\frac{v}{\|u\|^{0}}\right\|^{0}=\left\|\frac{w}{\|u\|^{0}}\right\|^{0}=1, \quad \frac{u}{\|u\|^{0}}=\frac{1}{2} \cdot \frac{v}{\|u\|^{0}}+\frac{1}{2} \cdot \frac{w}{\|u\|^{0}} .
$$

However, $\|u(t)\|=\|v(t)\|=\|w(t)\|$ for $t \in T$. By (b) of Theorem 2.4, we have $u=w$. Hence $v /\|u\|^{0}=w /\|u\|^{0}$. So $u$ is not an extreme point of $B\left(L_{M}^{0}(X)\right)$. Contradicting the rotundity of $L_{M}^{0}(X)$.

\section{Acknowledgment}

The authors would like to thank the anonymous referees for some suggestions to improve the paper. This work was supported by Heilong Jiang Natural Science Fund A200902.

\section{References}

[1] M. A. Smith, "A curious generalization of local uniform rotundity," Commentationes Mathematicae Universitatis Carolinae, vol. 25, no. 4, pp. 659-665, 1984.

[2] W. B. Moors and J. R. Giles, "Generic continuity of minimal set-valued mappings," Australian Mathematical Society A, vol. 63, no. 2, pp. 238-262, 1997.

[3] Z. Jimin, S. Lihuan, and C. Yun'an, "The HR-point of the Musielak-Orlicz function space equipped with Orlicz norm," Thai Journal of Mathematics, vol. 4, no. 1, pp. 209-222, 2006. 


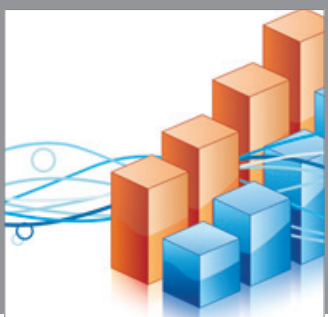

Advances in

Operations Research

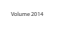

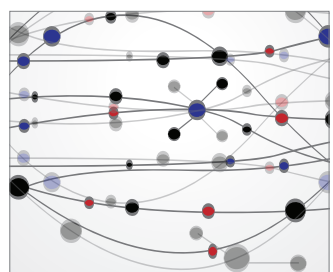

\section{The Scientific} World Journal
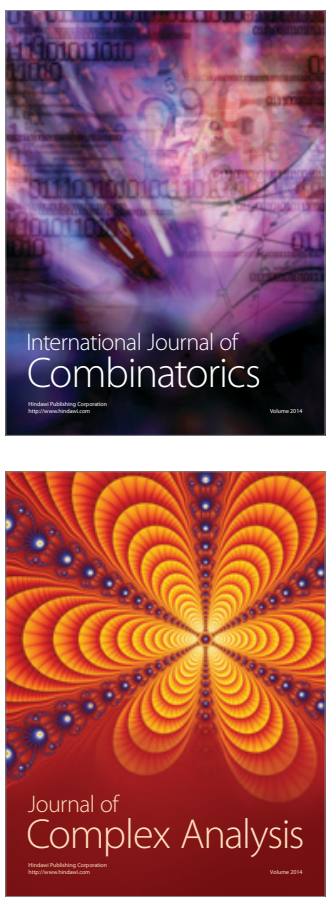

International Journal of

Mathematics and

Mathematical

Sciences
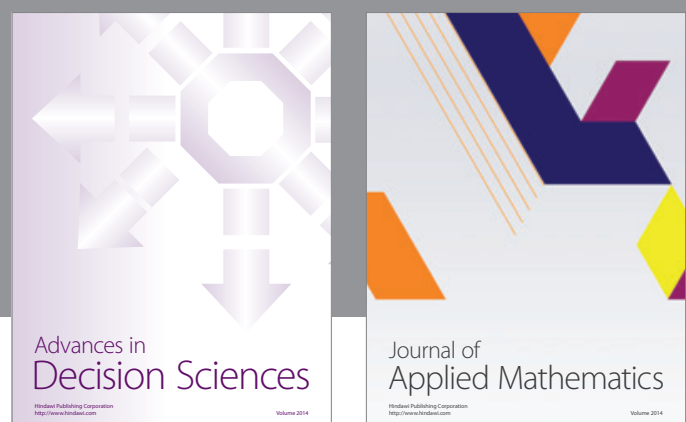

Journal of

Applied Mathematics
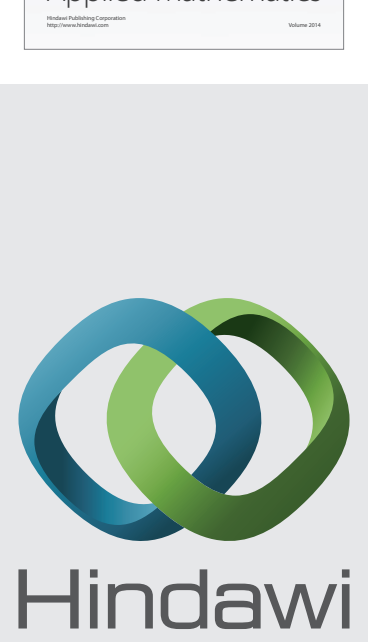

Submit your manuscripts at http://www.hindawi.com
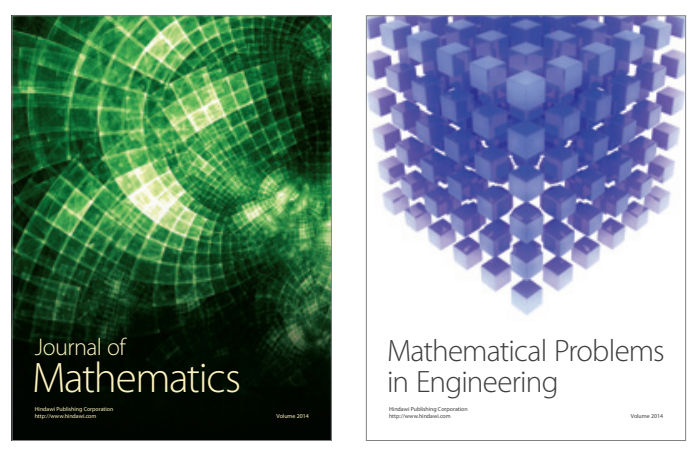

Mathematical Problems in Engineering
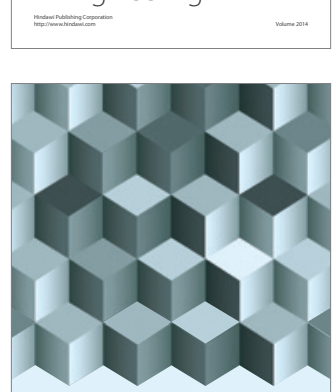

Journal of

Function Spaces
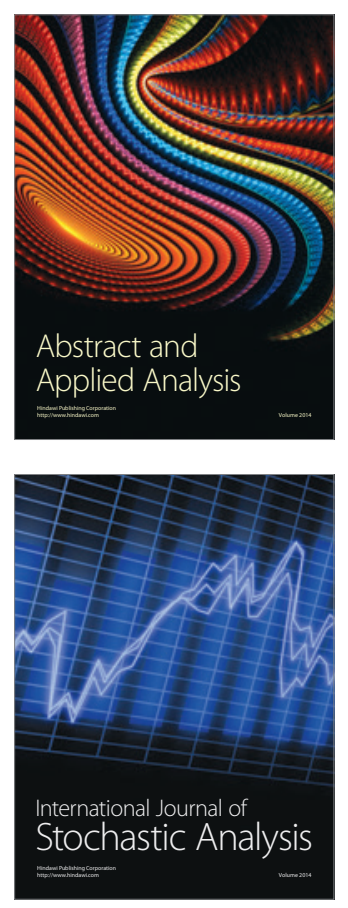

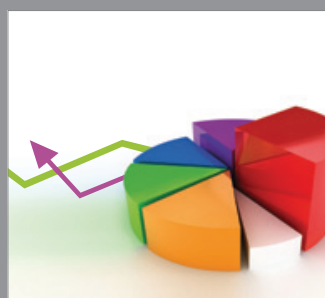

ournal of

Probability and Statistics

Promensencen
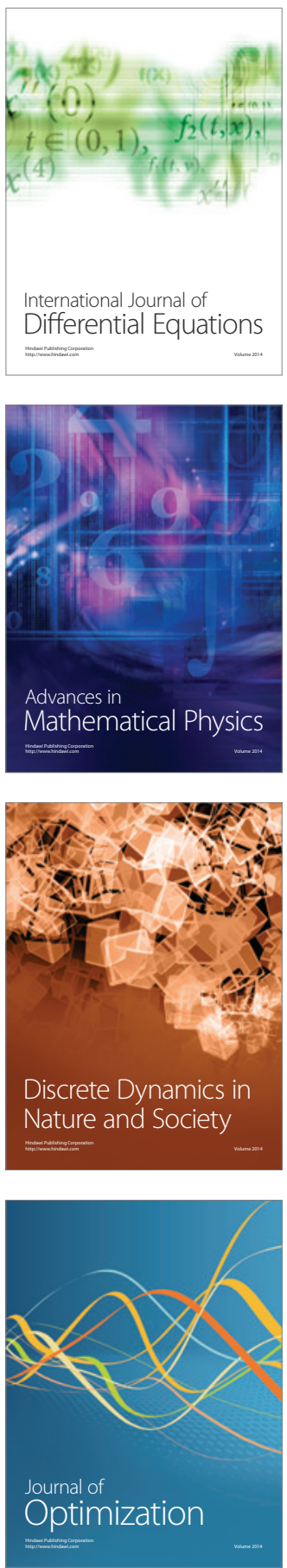